\title{
Null (Lightlike) $f$-Rectifying Curves in the Three Dimensional Minkowski Space $\mathbb{E}_{1}^{3}$
}

\author{
Zafar Iqbal $^{1^{*}}$ and Joydeep Sengupta ${ }^{2}$ \\ ${ }^{1}$ Department of Mathematics, Kaliyaganj College, Kaliyaganj, Uttar Dinajpur, West Bengal, INDIA \\ ${ }^{2}$ Department of Mathematics and Statistics, Aliah University, Newtown, Kolkata, West Bengal, INDIA \\ *Corresponding author
}

\section{Article Info}

Keywords: Curvature, Minkowski 3space, Null (lightlike) curve, Rectifying curve, Serret-Frenet formulae, Torsion 2010 AMS: 53A04, 53B30, 53C40, $53 C 50$

Received: 24 March 2020

Accepted: 15 May 2020

Available online: 10 June 2020

\begin{abstract}
A rectifying curve $\gamma$ in the Euclidean 3 -space $\mathbb{E}^{3}$ is defined as a space curve whose position vector always lies in its rectifying plane (i.e., the plane spanned by the unit tangent vector field $T_{\gamma}$ and the unit binormal vector field $B_{\gamma}$ of the curve $\gamma$ ), and an $f$-rectifying curve $\gamma$ in the Euclidean 3-space $\mathbb{E}^{3}$ is defined as a space curve whose $f$-position vector $\gamma_{f}$, defined by $\gamma_{f}(s)=\int f(s) d \gamma$, always lies in its rectifying plane, where $f$ is a nowhere vanishing real-valued integrable function in arc-length parameter $s$ of the curve $\gamma$. In this paper, we introduce the notion of $f$-rectifying curves which are null (lightlike) in the Minkowski 3 -space $\mathbb{E}_{1}^{3}$. Our main aim is to characterize and classify such null (lightlike) $f$-rectifying curves having spacelike or timelike rectifying plane in the Minkowski 3-Space $\mathbb{E}_{1}^{3}$.
\end{abstract}

\section{Introduction}

Let $\mathbb{E}^{3}$ denote the Euclidean 3-space. Let $\gamma: I \longrightarrow \mathbb{E}^{3}$ be a unit-speed curve parametrized by arc-length function $s$ with at least four continuous derivatives. Needless to mention, $I$ denotes a non-trivial interval in $\mathbb{R}$, i.e., a connected set in $\mathbb{R}$ containing at least two points. For the curve $\gamma$ in $\mathbb{E}^{3}$, we consider the Frenet apparatus $\left\{T_{\gamma}, N_{\gamma}, B_{\gamma}, \kappa_{\gamma}, \tau_{\gamma}\right\}$, where $T_{\gamma}$ is the unit tangent vector field, $N_{\gamma}$ is the unit principal normal vector field, $B_{\gamma}=T_{\gamma} \times N_{\gamma}$ is the unit binormal vector field of the curve $\gamma$, and $\kappa_{\gamma}: I \longrightarrow \mathbb{R}$ is a differentiable function with $\kappa_{\gamma}>0$, known as the curvature of $\gamma$, and $\tau_{\gamma}: I \longrightarrow \mathbb{R}$ is a differentiable function, called the torsion of $\gamma$. Then the Serret-Frenet formulae for the curve $\gamma$ are given by ([1]-[4])

$$
\left(\begin{array}{c}
T_{\gamma}^{\prime} \\
N_{\gamma}^{\prime} \\
B_{\gamma}^{\prime}
\end{array}\right)=\left(\begin{array}{ccc}
0 & \kappa_{\gamma} & 0 \\
-\kappa_{\gamma} & 0 & \tau_{\gamma} \\
0 & -\tau_{\gamma} & 0
\end{array}\right)\left(\begin{array}{c}
T_{\gamma} \\
N_{\gamma} \\
B_{\gamma}
\end{array}\right)
$$

The planes spanned by $\left\{T_{\gamma}, N_{\gamma}\right\},\left\{N_{\gamma}, B_{\gamma}\right\}$ and $\left\{T_{\gamma}, B_{\gamma}\right\}$ are called the osculating plane, the normal plane and the rectifying plane of the curve $\gamma$, respectively $([2,5])$.

In the Euclidean 3-space $\mathbb{E}^{3}$, the notion of a rectifying curve was introduced by B.Y. Chen in [5] as a tortuous curve whose position vector always lies in the rectifying plane of the curve. That is, for a rectifying curve $\gamma: I \longrightarrow \mathbb{E}^{3}$, the position vector of $\gamma$ can be expressed as

$$
\gamma(s)=\lambda(s) T_{\gamma}(s)+\mu(s) B_{\gamma}(s), s \in I
$$


for two differentiable functions $\lambda, \mu: I \longrightarrow \mathbb{R}$ in arc-length parameter $s$ of $\gamma$.

Several characterizations and classification of the rectifying curves in $\mathbb{E}^{3}$ were studied in [5]-[8]. Meanwhile the notion of rectifying curves were extended to several sort of Riemannian and pseudo-Riemannian spaces. As for example, many characterizations and classification of rectifying curves in the Minkowski 3-space $\mathbb{E}_{1}^{3}$ were studied in [9]-[11].

In this paper, we study null $f$-rectifying curves in the Minkowski 3-space $\mathbb{E}_{1}^{3}$. We organize this paper with three sections. In the first section, we give some basic preliminaries and then introduce the notion of $f$-rectifying curves which are null (or lightlike) in $\mathbb{E}_{1}^{3}$. Thereafter the second section is devoted to investigate some characterizations of null $f$-rectifying curves in $\mathbb{E}_{1}^{3}$. In the concluding section, we classify null $f$-rectifying curves in terms of their $f$-position vectors in $\mathbb{E}_{1}^{3}$.

\section{Preliminaries}

The Minkowski 3-space $\mathbb{E}_{1}^{3}$ is the Euclidean 3-space $\mathbb{E}^{3}$ equipped with the standard flat metric $g$ (called the Lorentzian inner product) defined by

$$
g(v, w)=v_{1} w_{1}+v_{2} w_{2}-v_{3} w_{3}
$$

for all tangent vectors $v=\left(v_{1}, v_{2}, v_{3}\right)$ and $w=\left(w_{1}, w_{2}, w_{3}\right)$ to $\mathbb{E}_{1}^{3}$ (see [12,13]). A tangent vector $v$ to $\mathbb{E}_{1}^{3}$ is called a

$$
\begin{aligned}
& \text { spacelike vector if and only if } g(v, v)>0 \quad \text { or } \quad v=0 \text {, } \\
& \text { lightlike vector (null vector) if and only if } g(v, v)=0 \text { and } v \neq 0 \text {, } \\
& \text { timelike vector if and only if } g(v, v)<0 \quad([12,13]) \text {. }
\end{aligned}
$$

As usual, the norm of a tangent vector $v$ to $\mathbb{E}_{1}^{3}$ is denoted and defined by $\|v\|=\sqrt{|g(v, v)|}$. It is trivial to mention that a tangent vector $v$ to $\mathbb{E}_{1}^{3}$ is called a unit vector if and only if $\|v\|=1$, i.e., if and only if $|g(v, v)|=1$, i.e., if and only if $g(v, v)= \pm 1$. Two tangent vectors $v$ and $w$ to $\mathbb{E}_{1}^{3}$ are said to be orthogonal if and only if $g(v, w)=0$. For any two tangent vectors $v$ and $w$ to $\mathbb{E}_{1}^{3}$, the vectorial product of $v$ and $w$ is defined by

$$
v \times w=\left|\begin{array}{ccc}
e_{1} & e_{2} & -e_{3} \\
v_{1} & v_{2} & v_{3} \\
w_{1} & w_{2} & w_{3}
\end{array}\right|=\left(v_{2} w_{3}-v_{3} w_{2}\right) e_{1}+\left(v_{3} w_{1}-v_{1} w_{3}\right) e_{2}+\left(v_{2} w_{1}-v_{1} w_{2}\right) e_{3}
$$

where $e_{i}=\left(\delta_{i 1}, \delta_{i 2}, \delta_{i 3}\right)$ for each $i \in\{1,2,3\}, \quad \delta_{i j}=\left\{\begin{array}{ll}1 & \text { if } i=j, \\ 0 & \text { if } i \neq j\end{array}\right.$ such that $e_{1} \times e_{2}=-e_{3}, e_{2} \times e_{3}=e_{1}, e_{3} \times e_{1}=$ $e_{2}([12,13])$.

Let $\gamma: I \longrightarrow \mathbb{E}_{1}^{3}$ be a curve in $\mathbb{E}_{1}^{3}$ and $\gamma^{\prime}$ stands for its velocity vector field. The curve $\gamma$ is said to be a spacelike curve, a lightlike curve (null curve) or a timelike curve in $\mathbb{E}_{1}^{3}$ if and only if its velocity vector $\gamma^{\prime}(t)$ is a spacelike vector, a lightlike vector (null vector) or a timelike vector, respectively, for each $t \in I$. To elaborate, the curve $\gamma$ in $\mathbb{E}_{1}^{3}$ is a

$$
\begin{aligned}
& \text { spacelike curve if and only if } g\left(\gamma^{\prime}(t), \gamma^{\prime}(t)\right)>0 \quad \text { or } \quad \gamma^{\prime}(t)=0 \text {, } \\
& \text { lightlike curve (null curve) if and only if } g\left(\gamma^{\prime}(t), \gamma^{\prime}(t)\right)=0 \text { and } \gamma^{\prime}(t) \neq 0 \text {, } \\
& \text { timelike curve if and only if } g\left(\gamma^{\prime}(t), \gamma^{\prime}(t)\right)<0
\end{aligned}
$$

for all $t \in I$ (see $[12,13]$ ). Thus, the curve $\gamma$ is said to be a non-null curve in $\mathbb{E}_{1}^{3}$ if and only if it is either a spacelike curve or a timelike curve in $\mathbb{E}_{1}^{3}$, i.e., if and only if $g\left(\gamma^{\prime}(t), \gamma^{\prime}(t)\right) \neq 0$ for all $t \in I$. If $\gamma$ is a non-null (spacelike or timelike) curve in $\mathbb{E}_{1}^{3}$ and we change the parameter $t$ by the function $s=s(t)$ given by $s(t)=\int_{0}^{t}\left\|\gamma^{\prime}(u)\right\| d u$ such that $\left\|\gamma^{\prime}(s)\right\|=\sqrt{\left|g\left(\gamma^{\prime}(s), \gamma^{\prime}(s)\right)\right|}=$ 1, i.e., $g\left(\gamma^{\prime}(s), \gamma^{\prime}(s)\right)= \pm 1$ for all $s \in I$, then the non-null curve $\gamma$ is said to be parametrized by arc-length function $s$ or a unitspeed non-null curve in $\mathbb{E}_{1}^{3}$. Again, if $\gamma$ is a null (lightlike) curve in $\mathbb{E}_{1}^{3}$ and we change the parameter $t$ by the function $s=s(t)$ given by $s(t)=\int_{0}^{t} \sqrt{\left\|\gamma^{\prime \prime}(u)\right\|} d u$ such that $g\left(\gamma^{\prime \prime}(s), \gamma^{\prime \prime}(s)\right)=1$ for all $s \in I$, then the null curve $\gamma$ is said to be parametrized by pseudo arc-length function $s$ or a unit-speed null curve in $\mathbb{E}_{1}^{3}$.

Let $\gamma: I \longrightarrow \mathbb{E}_{1}^{3}$ be a unit-speed null or non-null curve in the Minkowski 3-space $\mathbb{E}_{1}^{3}$ parametrized by arc-length function or pseudo arc-length function $s$ with Frenet apparatus $\left\{T_{\gamma}, N_{\gamma}, B_{\gamma}, \kappa_{\gamma}, \tau_{\gamma}\right\}$, where $\left\{T_{\gamma}, N_{\gamma}, B_{\gamma}=T_{\gamma} \times N_{\gamma}\right\}$ is the dynamic Frenet frame along the curve $\gamma$ in $\mathbb{E}_{1}^{3}$ and $\kappa_{\gamma}, \tau_{\gamma}$ are two differentiable functions in the parameter $s$ called, respectively, the curvature and the torsion of the curve $\gamma$ in $\mathbb{E}_{1}^{3}$. Then to write the Serret-Frenet formulae for the curve $\gamma$ the following mutually distinct cases come up for consideration:

Case I: Let $\gamma$ be a spacelike curve with a spacelike principal normal $N_{\gamma}$ in $\mathbb{E}_{1}^{3}$. Then the Serret-Frenet formulae for the curve $\gamma$ are given by

$$
\left(\begin{array}{c}
T_{\gamma}^{\prime} \\
N_{\gamma}^{\prime} \\
B_{\gamma}^{\prime}
\end{array}\right)=\left(\begin{array}{ccc}
0 & \kappa_{\gamma} & 0 \\
-\kappa_{\gamma} & 0 & \tau_{\gamma} \\
0 & \tau_{\gamma} & 0
\end{array}\right)\left(\begin{array}{c}
T_{\gamma} \\
N_{\gamma} \\
B_{\gamma}
\end{array}\right)
$$


where $g\left(T_{\gamma}(s), T_{\gamma}(s)\right)=1, \quad g\left(N_{\gamma}(s), N_{\gamma}(s)\right)=1, \quad g\left(B_{\gamma}(s), B_{\gamma}(s)\right)=-1, \quad g\left(T_{\gamma}(s), N_{\gamma}(s)\right)=0, \quad g\left(T_{\gamma}(s), B_{\gamma}(s)\right)=0$, $g\left(N_{\gamma}(s), B_{\gamma}(s)\right)=0$ for all $s \in I$.

Case II: Let $\gamma$ be a spacelike curve with a timelike principal normal $N_{\gamma}$ in $\mathbb{E}_{1}^{3}$. Then the Serret-Frenet formulae for the curve $\gamma$ are given by

$$
\left(\begin{array}{c}
T_{\gamma}^{\prime} \\
N_{\gamma}^{\prime} \\
B_{\gamma}^{\prime}
\end{array}\right)=\left(\begin{array}{ccc}
0 & \kappa_{\gamma} & 0 \\
\kappa_{\gamma} & 0 & \tau_{\gamma} \\
0 & \tau_{\gamma} & 0
\end{array}\right)\left(\begin{array}{c}
T_{\gamma} \\
N_{\gamma} \\
B_{\gamma}
\end{array}\right),
$$

where $g\left(T_{\gamma}(s), T_{\gamma}(s)\right)=1, \quad g\left(N_{\gamma}(s), N_{\gamma}(s)\right)=-1, \quad g\left(B_{\gamma}(s), B_{\gamma}(s)\right)=1, \quad g\left(T_{\gamma}(s), N_{\gamma}(s)\right)=0, g\left(T_{\gamma}(s), B_{\gamma}(s)\right)=0$, $g\left(N_{\gamma}(s), B_{\gamma}(s)\right)=0$ for all $s \in I$.

Case III: Let $\gamma$ be a spacelike curve with a null principal normal $N_{\gamma}$ in $\mathbb{E}_{1}^{3}$. Then the Serret-Frenet formulae for the curve $\gamma$ are given by

$$
\left(\begin{array}{c}
T_{\gamma}^{\prime} \\
N_{\gamma}^{\prime} \\
B_{\gamma}^{\prime}
\end{array}\right)=\left(\begin{array}{ccc}
0 & \kappa_{\gamma} & 0 \\
0 & \tau_{\gamma} & 0 \\
-\kappa_{\gamma} & 0 & -\tau_{\gamma}
\end{array}\right)\left(\begin{array}{c}
T_{\gamma} \\
N_{\gamma} \\
B_{\gamma}
\end{array}\right),
$$

where $g\left(T_{\gamma}(s), T_{\gamma}(s)\right)=1, \quad g\left(N_{\gamma}(s), N_{\gamma}(s)\right)=0, \quad g\left(B_{\gamma}(s), B_{\gamma}(s)\right)=0, \quad g\left(T_{\gamma}(s), N_{\gamma}(s)\right)=0, \quad g\left(T_{\gamma}(s), B_{\gamma}(s)\right)=0$, $g\left(N_{\gamma}(s), B_{\gamma}(s)\right)=1$ for all $s \in I$. In this case, $\kappa_{\gamma}$ can take only two values: $\kappa_{\gamma}=0$ if $\gamma$ is a straight line and $\kappa_{\gamma}=1$ in the remaining cases.

Case IV: Let $\gamma$ be a timelike curve in $\mathbb{E}_{1}^{3}$. Then the Serret-Frenet formulae for the curve $\gamma$ are given by

$$
\left(\begin{array}{c}
T_{\gamma}^{\prime} \\
N_{\gamma}^{\prime} \\
B_{\gamma}^{\prime}
\end{array}\right)=\left(\begin{array}{ccc}
0 & \kappa_{\gamma} & 0 \\
\kappa_{\gamma} & 0 & \tau_{\gamma} \\
0 & -\tau_{\gamma} & 0
\end{array}\right)\left(\begin{array}{c}
T_{\gamma} \\
N_{\gamma} \\
B_{\gamma}
\end{array}\right),
$$

where $g\left(T_{\gamma}(s), T_{\gamma}(s)\right)=-1, \quad g\left(N_{\gamma}(s), N_{\gamma}(s)\right)=1, \quad g\left(B_{\gamma}(s), B_{\gamma}(s)\right)=1, \quad g\left(T_{\gamma}(s), N_{\gamma}(s)\right)=0, \quad g\left(T_{\gamma}(s), B_{\gamma}(s)\right)=0$, $g\left(N_{\gamma}(s), B_{\gamma}(s)\right)=0$ for all $s \in I$.

Case V: Let $\gamma$ be a null (lightlike) curve in $\mathbb{E}_{1}^{3}$. Then the Serret-Frenet formulae for the curve $\gamma$ are given by

$$
\left(\begin{array}{c}
T_{\gamma}^{\prime} \\
N_{\gamma}^{\prime} \\
B_{\gamma}^{\prime}
\end{array}\right)=\left(\begin{array}{ccc}
0 & \kappa_{\gamma} & 0 \\
\tau_{\gamma} & 0 & -\kappa_{\gamma} \\
0 & -\tau_{\gamma} & 0
\end{array}\right)\left(\begin{array}{c}
T_{\gamma} \\
N_{\gamma} \\
B_{\gamma}
\end{array}\right),
$$

where $g\left(T_{\gamma}(s), T_{\gamma}(s)\right)=0, \quad g\left(N_{\gamma}(s), N_{\gamma}(s)\right)=1, \quad g\left(B_{\gamma}(s), B_{\gamma}(s)\right)=0, \quad g\left(T_{\gamma}(s), N_{\gamma}(s)\right)=0, \quad g\left(T_{\gamma}(s), B_{\gamma}(s)\right)=1$, $g\left(N_{\gamma}(s), B_{\gamma}(s)\right)=0$ for all $s \in I$. In this case, $\kappa_{\gamma}$ can take only two values: $\kappa_{\gamma}=0$ if $\gamma$ is a straight null line and $\kappa_{\gamma}=1$ in the remaining cases.

The two-dimensional pseudo-Riemannian sphere of unit radius and centred at the origin in $\mathbb{E}_{1}^{3}$ is denoted and defined by

$$
\mathbb{S}_{1}^{2}(1):=\left\{v \in \mathbb{E}_{1}^{3}: g(v, v)=1\right\},
$$

and the two-dimensional pseudo-hyperbolic space of unit radius and centred at the origin in $\mathbb{E}_{1}^{3}$ is denoted and defined by

$$
\mathbb{H}_{0}^{2}(1):=\left\{v \in \mathbb{E}_{1}^{3}: g(v, v)=-1\right\} .
$$

For more elaborations of the above discussion please see [9]-[13].

An arbitrary plane $\pi$ in $\mathbb{E}_{1}^{3}$ is spacelike, timelike or lightlike if the induced Lorentzian metric $\left.g\right|_{\pi}$ is respectively positive definite, non-degenerate of index 1 , or degenerate. A unit-speed null curve $\gamma: I \longrightarrow \mathbb{E}_{1}^{3}$ parametrized by pseudo arc-length function $s$ is called a rectifying curve in $\mathbb{E}_{1}^{3}$ if its position vector always lies in its rectifying plane in $\mathbb{E}_{1}^{3}$, i.e., if its position vector $\gamma$ in $\mathbb{E}_{1}^{3}$ can be expressed as

$$
\gamma(s)=\lambda(s) T_{\gamma}(s)+\mu(s) B_{\gamma}(s), s \in I,
$$

for some differentiable functions $\lambda, \mu: I \longrightarrow \mathbb{R}$ in pseudo arc-length parameter $s$ of $\gamma$. Now, for some non-zero integrable function $f: I \longrightarrow \mathbb{R}$ in pseudo arc-length function $s$, the $f$-position vector of the curve $\gamma$ in $\mathbb{E}_{1}^{3}$ is denoted by $\gamma_{f}$ and is defined by

$$
\gamma_{f}(s):=\int f(s) d \gamma
$$

for all $s \in I$. Keeping in mind this notion of position vector of a curve in $\mathbb{E}_{1}^{3}$, we define a null $f$-rectifying curve in $\mathbb{E}_{1}^{3}$ as follows: 
Definition 2.1. (Null $f$-Rectifying Curve) Let $\gamma: I \longrightarrow \mathbb{E}_{1}^{3}$ be a unit-speed null curve in $\mathbb{E}_{1}^{3}$ parametrized by pseudo arc-length function s with Frenet apparatus $\left\{T_{\gamma}, N_{\gamma}, B_{\gamma}, \kappa_{\gamma}, \tau_{\gamma}\right\}$, and let $f: I \longrightarrow \mathbb{R}$ be a nowhere vanishing integrable function in pseudo arc-length parameter $s$. The curve $\gamma$ is called an $f$-rectifying curve in $\mathbb{E}_{1}^{3}$ if its $f$-position vector $\gamma_{f}=\int f d \gamma$ always lies in its rectifying plane in $\mathbb{E}_{1}^{3}$, i.e., if its $f$-position vector $\gamma_{f}=\int f d \gamma$ in $\mathbb{E}_{1}^{3}$ can be expressed as

$$
\gamma_{f}(s)=\int f(s) d \gamma=\lambda(s) T_{\gamma}(s)+\mu(s) B_{\gamma}(s), s \in I,
$$

for two differentiable functions $\lambda, \mu: I \longrightarrow \mathbb{R}$ in pseudo arc-length parameter $s$.

In the next section, we shall see that if the function $f$ vanishes on $I$, then the ratio $\frac{\tau_{\gamma}}{\kappa_{\gamma}}$ for the curve $\gamma$ in $\mathbb{E}_{1}^{3}$ is constant, and hence it becomes a helix in $\mathbb{E}_{1}^{3}$. This is why we have taken here the function $f$ as nowhere vanishing integrable function on $I$. And if the function $f$ is a non-zero constant on $I$, then the ratio $\frac{\tau_{\gamma}}{\kappa_{\gamma}}$ for the curve $\gamma$ in $\mathbb{E}_{1}^{3}$ is a non-constant linear function in pseudo arc-length parameter $s$, and hence it reduces to a rectifying curve in $\mathbb{E}_{1}^{3}$.

\section{Characterizations of null $f$-rectifying curves in the Minkowski 3 -space $\mathbb{E}_{1}^{3}$}

First, we mention (and then prove) a theorem in which we characterize unit-speed null (lightlike) $f$-rectifying curves in the Minkowski 3-space $\mathbb{E}_{1}^{3}$ in terms of the norm functions, tangential components and binormal components of their $f$-position vectors.

Theorem 3.1. Let $\gamma: I \longrightarrow \mathbb{E}_{1}^{3}$ be a unit-speed null $f$-rectifying curve in $\mathbb{E}_{1}^{3}$ parametrized by pseudo arc-length function $s$ with the curvature function $\kappa_{\gamma} \equiv 1$ and the torsion function $\tau_{\gamma}$, and let $f: I \longrightarrow \mathbb{R}$ be a nowhere vanishing integrable function in pseudo arc-length parameter $s$ with primitive function $F$. Then the following statements hold:

1. The norm function $\rho=\left\|\gamma_{f}\right\|$ is given by

$$
\rho(s)=\sqrt{|2 c F(s)|}
$$

for all $s \in I$, where $c$ is a non-zero constant.

2. The tangential component $g\left(\gamma_{f}, T_{\gamma}\right)$ of the $f$-position vector $\gamma_{f}$ of the curve $\gamma$ is a non-zero constant.

3. The torsion function $\tau_{\gamma}$ is non-zero, and the binormal component $g\left(\gamma_{f}, B_{\gamma}\right)$ of the $f$-position vector $\gamma_{f}$ of the curve $\gamma$ is given by

$$
g\left(\gamma_{f}(s), B_{\gamma}(s)\right)=F(s)=\int f(s) d s
$$

for all $s \in I$.

Conversely, if $f: I \longrightarrow \mathbb{R}$ is a nowhere vanishing integrable function in pseudo arc-length function $s$ with primitive function $F$, and if $\gamma: I \longrightarrow \mathbb{E}_{1}^{3}$ is a unit-speed null curve in $\mathbb{E}_{1}^{3}$ and with the curvature function $\kappa_{\gamma} \equiv 1$ and the torsion function $\tau_{\gamma}$, and any one of the statements 1,2 or 3 holds, then $\gamma$ is an $f$-rectifying curve in $\mathbb{E}_{1}^{3}$.

Proof. Let us first assume that $\gamma: I \longrightarrow \mathbb{E}_{1}^{3}$ be a unit-speed null $f$-rectifying curve in $\mathbb{E}_{1}^{3}$ parametrized by pseudo arc-length function $s$ with the curvature function $\kappa_{\gamma} \equiv 1$ and the torsion function $\tau_{\gamma}$, where $f: I \longrightarrow \mathbb{R}$ is a nowhere vanishing integrable function in pseudo arc-length parameter $s$ with primitive function $F$. Then the $f$-position vector $\gamma_{f}$ of the curve $\gamma$ can be expressed as

$$
\gamma_{f}(s)=\int f(s) d \gamma=\lambda(s) T_{\gamma}(s)+\mu(s) B_{\gamma}(s), s \in I
$$

for two derivable functions $\lambda, \mu: I \longrightarrow \mathbb{R}$ in pseudo arc-length parameter $s$. Differentiating both the sides of the equation (3.1) with respect to $s$ and then applying the Serret-Frenet formulae (2.1), we obtain

$$
f(s) T_{\gamma}(s)=\lambda^{\prime}(s) T_{\gamma}(s)+\left(\lambda(s)-\mu(s) \tau_{\gamma}(s)\right) N_{\gamma}(s)+\mu^{\prime}(s) B_{\gamma}(s)
$$

for all $s \in I$. Equating the coefficients of like-terms from both the sides of equation (3.2), we find

$$
\lambda^{\prime}(s)=f(s), \quad \lambda(s)-\mu(s) \tau_{\gamma}(s)=0, \quad \mu^{\prime}(s)=0
$$


which implies

$$
\left\{\begin{array}{l}
\lambda(s)=\int f(s) d s=F(s) \\
\tau_{\gamma}(s)=\frac{\lambda(s)}{\mu(s)} \\
\mu(s)=\text { a non-zero constant }=c \text { (suppose) }
\end{array}\right.
$$

for all $s \in I$. We have the following:

1. Using the equation (3.1) and the relations (3.3), the norm function $\rho=\left\|\gamma_{f}\right\|$ is given by

$$
\rho^{2}(s)=\left\|\gamma_{f}(s)\right\|^{2}=\left|g\left(\gamma_{f}(s), \gamma_{f}(s)\right)\right|=|2 c F(s)|
$$

for all $s \in I$. That is,

$$
\rho(s)=\sqrt{|2 c F(s)|}
$$

for all $s \in I$, where $c$ is a non-zero constant.

2. Using the equation (3.1) and the relations (3.3), the tangential component $g\left(\gamma_{f}, T_{\gamma}\right)$ of the $f$-position vector $\gamma_{f}$ of $\gamma$ is given by

$$
g\left(\gamma_{f}(s), T_{\gamma}(s)\right)=\mu(s)=c
$$

for all $s \in I$. Hence, the tangential component $g\left(\gamma_{f}, T_{\gamma}\right)$ of the $f$-position vector $\gamma_{f}$ of the curve $\gamma$ is a non-zero constant.

3. From the relations (3.3) it is evident that $\tau_{\gamma}(s) \neq 0$ for all $s \in I$. Using the equation (3.1) and the relations (3.3), the binormal component $g\left(\gamma_{f}, B_{\gamma}\right)$ of the $f$-position vector $\gamma_{f}$ of $\gamma$ is given by

$$
g\left(\gamma_{f}(s), B_{\gamma}(s)\right)=\lambda(s)=F(s)
$$

for all $s \in I$.

Conversely, we assume that $f: I \longrightarrow \mathbb{R}$ is a nowhere vanishing integrable function in pseudo arc-length function $s$ with primitive function $F$, and we also assume that $\gamma: I \longrightarrow \mathbb{E}_{1}^{3}$ is a unit-speed null (lightlike) curve in $\mathbb{E}_{1}^{3}$ and with the curvature function $\kappa_{\gamma} \equiv 1$ and the torsion function $\tau_{\gamma}$, and the statement 1 or 2 holds. For the statement 1 , we have

$$
g\left(\gamma_{f}(s), \gamma_{f}(s)\right)=2 c F(s)
$$

for all $s \in I$, where $c$ is a non-zero constant. Differentiating both the sides of the equation (3.4), and using the relations $\gamma_{f}^{\prime}(s)=f(s) T_{\gamma}(s)$ and $F^{\prime}(s)=f(s)$ for all $s \in I$, we obtain

$$
g\left(\gamma_{f}(s), T(s)\right)=c
$$

for all $s \in I$. This is nothing but the statement 2. So, in either case, we find the equation (3.5). Now, differentiating both the sides of the equation (3.5) with respect to $s$, and applying the relations $\gamma_{f}^{\prime}(s)=f(s) T_{\gamma}(s), T_{\gamma}^{\prime}(s)=\kappa_{\gamma}(s) N_{\gamma}(s), \kappa_{\gamma}(s)=1$ and $g\left(T_{\gamma}(s), T_{\gamma}(s)\right)=0$ for all $s \in I$, we obtain

$$
\begin{aligned}
f(s) g\left(T_{\gamma}(s), T_{\gamma}(s)\right)+\kappa_{\gamma}(s) g\left(\gamma_{f}(s), N_{\gamma}(s)\right) & =0 \\
g\left(\gamma_{f}(s), N_{\gamma}(s)\right) & =0
\end{aligned}
$$

for all $s \in I$. This asserts us that $\gamma$ is an $f$-rectifying curve in $\mathbb{E}_{1}^{3}$.

Finally, we assume that the statement 3 holds. Then for all $s \in I$, we have

$$
g\left(\gamma_{f}(s), B_{\gamma}(s)\right)=F(s)
$$

Differentiating both the sides of the equation (3.6) with respect to $s$, and in virtue of the relations $\gamma_{f}^{\prime}(s)=f(s) T_{\gamma}(s), B_{\gamma}^{\prime}(s)=$ $-\tau_{\gamma}(s) N_{\gamma}(s), \tau_{\gamma}(s) \neq 0, g\left(T_{\gamma}(s), B_{\gamma}(s)\right)=1$ and $F^{\prime}(s)=f(s)$ for all $s \in I$, we obtain

$$
\begin{aligned}
f(s) g\left(T_{\gamma}(s), B_{\gamma}(s)\right)-\tau_{\gamma}(s) g\left(\gamma_{f}(s), N_{\gamma}(s)\right) & =f(s) \\
g\left(\gamma_{f}(s), N_{\gamma}(s)\right) & =0
\end{aligned}
$$

for all $s \in I$. This asserts us that $\gamma$ is an $f$-rectifying curve in $\mathbb{E}_{1}^{3}$. 
In the next theorem, we characterize a unit-speed null $f$-rectifying curve in the Minkowski 3-space $\mathbb{E}_{1}^{3}$ by virtue of the ratio $\frac{\tau_{\gamma}}{\kappa_{\gamma}}$ of the curvature function $\kappa_{\gamma}$ and the torsion function $\tau_{\gamma}$.

Theorem 3.2. Let $\gamma: I \longrightarrow \mathbb{E}_{1}^{3}$ be a unit-speed null curve in $\mathbb{E}_{1}^{3}$ parametrized by pseudo arc-length function $s$ with the curvature function $\kappa_{\gamma} \equiv 1$ and the torsion function $\tau_{\gamma}$. Also, let $f: I \longrightarrow \mathbb{R}$ be a nowhere vanishing integrable function in pseudo arc-length parameter $s$ with primitive function $F$. Then, up to isometries of $\mathbb{E}_{1}^{3}$, the curve $\gamma$ is congruent to an $f$-rectifying curve in $\mathbb{E}_{1}^{3}$ if and only if the ratio $\frac{\tau_{\gamma}}{\kappa_{\gamma}}$ satisfies

$$
\frac{\tau_{\gamma}(s)}{\kappa_{\gamma}(s)}=\frac{1}{c} F(s)
$$

for all $s \in I$, where $c$ is a non-zero constant.

Proof. Let us first assume that $\gamma: I \longrightarrow \mathbb{E}_{1}^{3}$ be a unit-speed null $f$-rectifying curve in $\mathbb{E}_{1}^{3}$ parametrized by pseudo arc-length function $s$ with the curvature function $\kappa_{\gamma} \equiv 1$ and the torsion function $\tau_{\gamma}$, and $f: I \longrightarrow \mathbb{R}$ is a nowhere vanishing integrable function in pseudo arc-length parameter $s$ with primitive function $F$. Then from the second one of the relations (3.3), we have

$$
\frac{\tau_{\gamma}(s)}{\kappa_{\gamma}(s)}=\frac{\lambda(s)}{\mu(s)}=\frac{1}{c} F(s)
$$

for all $s \in I$, where $c$ is a non-zero constant.

Conversely, we assume that $\gamma: I \longrightarrow \mathbb{E}_{1}^{3}$ be a unit-speed null curve in $\mathbb{E}_{1}^{3}$ parametrized $s$ with the curvature function $\kappa_{\gamma} \equiv 1$ and the torsion function $\tau_{\gamma}$, where $f: I \longrightarrow \mathbb{R}$ is a nowhere vanishing integrable function in pseudo arc-length parameter $s$ with primitive function $F$ such that the ratio $\frac{\tau_{\gamma}}{\kappa_{\gamma}}$ is given by

$$
\frac{\tau_{\gamma}(s)}{\kappa_{\gamma}(s)}=\frac{1}{c} F(s)
$$

for all $s \in I$, where $c$ is a non-zero constant. Then by applying the Serret-Frenet formulae (2.1), we obtain

$$
\frac{d}{d s}\left(\gamma_{f}(s)-F(s) T_{\gamma}(s)-c B_{\gamma}(s)\right)=0
$$

for all $s \in I$. This proves that, up to isometries of $\mathbb{E}_{1}^{3}, \gamma$ is an $f$-rectifying curve in $\mathbb{E}_{1}^{3}$.

Remark 3.3. Let $\gamma: I \longrightarrow \mathbb{E}_{1}^{3}$ be a unit-speed null curve in $\mathbb{E}_{1}^{3}$ parametrized by pseudo arc-length function $s$ with curvature function $\kappa_{\gamma} \equiv 1$ and the torsion function $\tau_{\gamma}$. If the function $f$ vanishes identically on $I$, then its primitive function $F$ is a constant on I. Hence, by the previous theorem, the ratio $\frac{\tau_{\gamma}}{\kappa_{\gamma}}$ for the curve $\gamma$ in $\mathbb{E}_{1}^{3}$ is given by

$$
\frac{\tau_{\gamma}(s)}{\kappa_{\gamma}(s)}=\frac{1}{c} F(s)=\text { a constant }
$$

for all $s \in I$. Consequently, the curve $\gamma$ reduces to becomes a helix in $\mathbb{E}_{1}^{3}$ ([1]).

Again, if the function $f$ is a non-zero constant on $I$, then its primitive function $F$ is given by

$$
F(s)=c_{1} s+c_{2}
$$

for all $s \in I$, where $c_{1}$ and $c_{2}$ are constants. Hence, by the previous theorem, the ratio $\frac{\tau_{\gamma}}{\kappa_{\gamma}}$ for the curve $\gamma$ in $\mathbb{E}_{1}^{3}$ is given by

$$
\frac{\tau_{\gamma}(s)}{\kappa_{\gamma}(s)}=\frac{1}{c} F(s)=\frac{1}{c}\left(c_{1} s+c_{2}\right)=a s+b
$$

for all $s \in I$, where $a=\frac{c_{1}}{c}(\neq 0)$ and $b=\frac{c_{2}}{c}$ are constants. Thus, the ratio $\frac{\tau_{\gamma}}{\kappa_{\gamma}}$ is a non-constant linear function in pseudo arc-length parameter $s$. Consequently, the curve $\gamma$ reduces to a rectifying curve in $\mathbb{E}_{1}^{3}([11])$. 


\section{Classification of null $f$-rectifying curves in the Minkowski 3-space $\mathbb{E}_{1}^{3}$}

In this section, we determine explicitly all unit-speed null $f$-rectifying curves in the Minkowski 3 -space $\mathbb{E}_{1}^{3}$ in terms of their $f$-position vectors. The main theorem reads as follows:

Theorem 4.1. Let $\gamma: I \longrightarrow \mathbb{E}_{1}^{3}$ be a unit-speed null curve in $\mathbb{E}_{1}^{3}$ parametrized by pseudo arc-length function $s$ and $f: I \longrightarrow \mathbb{R}$ be a nowhere vanishing integrable function in s with primitive function $F$. Then $\gamma$ is an $f$-rectifying curve in $\mathbb{E}_{1}^{3}$ having a spacelike (or timelike) $f$-position vector $\gamma_{f}$ if and only if, up to a parametrization, its $f$-position vector $\gamma_{f}$ is given by

$$
\gamma_{f}(t)=\sqrt{2 c F(0)} e^{t} y(t)
$$

for all possible $t$, where $c$ is a positive constant, $F(0)>0$ and $y=y(t)$ is a unit-speed timelike (respectively spacelike) curve in the pseudo-sphere $\mathbb{S}_{1}^{2}(1)$ (respectively the pseudo-hyperbolic space $\mathbb{H}_{0}^{2}(1)$ ).

Proof. First, we assume that $\gamma$ is a unit-speed null $f$-rectifying curve in $\mathbb{E}_{1}^{3}$ having a spacelike $f$-position vector $\gamma_{f}$, where $f: I \longrightarrow \mathbb{R}$ is a nowhere vanishing integrable function in $s$ with primitive function $F$. Then we have

$$
g\left(\gamma_{f}(s), \gamma_{f}(s)\right)>0, \quad g\left(T_{\gamma}(s), T_{\gamma}(s)\right)=0
$$

for all $s \in I$, and from the proof of the Theorem 3.1, we obtain

$$
\rho^{2}(s)=\left\|\gamma_{f}(s)\right\|^{2}=\left|g\left(\gamma_{f}(s), \gamma_{f}(s)\right)\right|=2 c F(s),
$$

for all $s \in I$, where we may choose $c$ as an arbitrary positive constant. Now, we define a curve $y=y(s)$ by

$$
y(s):=\frac{\gamma_{f}(s)}{\rho(s)}
$$

for all $s \in I$. Then we have

$$
g(y(s), y(s))=\frac{g\left(\gamma_{f}(s), \gamma_{f}(s)\right)}{\rho^{2}(s)}=1,
$$

for all $s \in I$. Therefore, $y=y(s)$ is a curve in the pseudo-sphere $\mathbb{S}_{1}^{2}(1)$. Differentiating both the sides of the equation (4.3) with respect to $s$, we obtain

$$
g\left(y(s), y^{\prime}(s)\right)=0
$$

for all $s \in I$. Now, from the equations (4.1) and (4.2), we find

$$
\gamma_{f}(s)=y(s) \sqrt{2 c F(s)}
$$

for all $s \in I$. Differentiating both the sides of the equation (4.5) with respect to $s$, we get

$$
f(s) T_{\gamma}(s)=y^{\prime}(s) \sqrt{2 c F(s)}+\frac{c f(s) y(s)}{\sqrt{2 c F(s)}},
$$

for all $s \in I$. From the equations (4.3), (4.4) and (4.6), we obtain

$$
g\left(y^{\prime}(s), y^{\prime}(s)\right)=-\frac{f^{2}(s)}{4 F^{2}(s)}
$$

for all $s \in I$. This indicates that $y$ is a timelike curve. From the equation (4.7), we find

$$
\left\|y^{\prime}(s)\right\|=\sqrt{\left|g\left(y^{\prime}(s), y^{\prime}(s)\right)\right|}=\frac{f(s)}{2 F(s)}
$$

for all $s \in I$. Let $t$ be arc-length parameter of the curve $y$ in $\mathbb{S}_{1}^{2}(1)$ given by

$$
t=\int_{0}^{s}\left\|y^{\prime}(u)\right\| d u .
$$

Then we obtain

$$
\begin{aligned}
t & =\int_{0}^{s} \frac{f(u)}{2 F(u)} d u \\
\Longrightarrow \quad t & =\frac{1}{2} \ln F(s)-\frac{1}{2} \ln F(0) \\
\Longrightarrow \quad F(s) & =F(0) e^{2 t}
\end{aligned}
$$


It is obvious that $F(0)>0$. Substituting the result (4.8) in (4.5), we obtain the $f$-position vector of $\gamma$ as follows:

$$
\gamma_{f}(t)=y(t) \sqrt{2 c F(0) e^{2 t}}=\sqrt{2 c F(0)} e^{t} y(t)
$$

for all possible $t$, where $c$ is a positive constant, $F(0)>0$ and $y=y(t)$ is a unit-speed timelike curve in the pseudo-sphere $\mathbb{S}_{1}^{2}(1)$

Conversely, we assume that $\gamma$ is a unit-speed null curve in $\mathbb{E}_{1}^{3}$ such that for some nowhere vanishing integrable function $f: I \longrightarrow \mathbb{R}$ in $s$ with primitive function $F$ the $f$-position vector $\gamma_{f}$ of $\gamma$ is given by

$$
\gamma_{f}(t):=\sqrt{2 c F(0)} e^{t} y(t)
$$

for all possible $t$, where $c$ is a positive constant, $F(0)>0$ and $y=y(t)$ is a unit-speed timelike curve in the pseudo-sphere $\mathbb{S}_{1}^{2}(1)$. Since $y=y(t)$ is a unit-speed timelike curve in the pseudo-sphere $\mathbb{S}_{1}^{2}(1)$, we have $g\left(y^{\prime}(t), y^{\prime}(t)\right)=-1, g(y(t), y(t))=1$ and consequently $g\left(y(t), y^{\prime}(t)\right)=0$ for all $t$. Therefore, from the equation (4.9), we have

$$
g\left(\gamma_{f}(t), \gamma_{f}(t)\right)=2 c F(0) e^{2 t}
$$

for all $t$. Now, we may reparametrize the curve $\gamma$ by

$$
t=\frac{1}{2}(\ln F(s)-\ln F(0)),
$$

where $s$ stands for arc-length parameter of $\gamma$. Then from (4.10), we have

$$
g\left(\gamma_{f}(s), \gamma_{f}(s)\right)=2 c F(s)
$$

for all $s \in I$. Therefore, the norm function $\rho=\left\|\gamma_{f}\right\|$ is given by

$$
\rho^{2}(s)=\left\|\gamma_{f}(s)\right\|^{2}=\left|g\left(\gamma_{f}(s), \gamma_{f}(s)\right)\right|=|2 c F(s)|
$$

for all $s \in I$, that is,

$$
\rho(s)=\sqrt{|2 c F(s)|}
$$

for all $s \in I$, where $c$ is a positive constant. Therefore, by applying Theorem 3.1, we conclude the nature of $\gamma$ as an $f$-rectifying curve in $\mathbb{E}_{1}^{3}$.

The proof is analogous when $\gamma$ is considered as a unit-speed null $f$-rectifying curve in $\mathbb{E}_{1}^{3}$ having a timelike $f$-position vector $\gamma_{f}$.

\section{Conclusion}

In this paper, we introduced the notion of null (lightlike) $f$-rectifying curves in the Minkowski 3-Space $\mathbb{E}_{1}^{3}$ for some nowhere vanishing integrable function $f: I \longrightarrow \mathbb{R}$ in pseudo arc-length parameter $s$ with primitive function $F$. Then we characterized such curves in $\mathbb{E}_{1}^{3}$. In Theorem 3.1, we have shown that for a unit-speed $f$-rectifying curve $\gamma$ in $\mathbb{E}_{1}^{3}$, the norm function of its $f$-position vector $\gamma_{f}$ is expressed in terms of the primitive function $F$, the tangential component of its $f$-position vector $\gamma_{f}$ is a non-zero constant and the binormal component of its $f$-position vector $\gamma_{f}$ is nothing but the primitive function $F$. Thereafter, in Theorem 3.2, it is shown that for a unit-speed $f$-rectifying curve $\gamma$ in $\mathbb{E}_{1}^{3}$, the ratio $\frac{\tau_{\gamma}}{\kappa_{\gamma}}$ of the curvature function $\kappa_{\gamma}$ and the torsion function $\tau_{\gamma}$ is a non-zero constant multiple of the primitive function $F$. Finally, in Theorem 4.1, we classified all such unit-speed null $f$-rectifying curves having spacelike or timelike $f$-position vectors in $\mathbb{E}_{1}^{3}$.

\section{Acknowledgement}

We would like to express our heartiest gratitude to Prof. Subenoy Chakraborty, Professor, Department of Mathematics, Jadavpur University, INDIA, for guiding us with his pearls of wisdom during the preparation of this article. We are also grateful to the anonymous honourable referees for their valuable time dedicated to this article and for their thorough comments which helped to improve this article.

\section{References}

[1] A. Pressley, Elementary Differential Geometry, 2nd ed., Springer, 2010.

[2] M. P. do Carmo, Differential Geometry of Curves and Surfaces: Revised and Updated Second Edition, Courier Dover Publications, 2016.

[3] M. Spivak, A Comprehensive Introduction to Differential Geometry, Vol. 2, 3rd ed., Publish or Perish, Houston, Texas, 1999.

[4] R. S. Millman, G. D. Parker, Elements of Differential Geometry, Prentice-Hall, Inc., New Jersey, 1977. 
[5] B. Y. Chen, When does the position vector of a space curve always lie in its rectifying plane?, Amer. Math. Monthly, 110 (2003), 147-152.

[6] B. Y. Chen, Rectifying curves and geodesics on a cone in the Euclidean 3-space, Tamkang J. Math., 48 (2017), $209-214$.

[7] B. Y. Chen, F. Dillen, Rectifying curves as centrodes and extremal curves, Bull. Inst. Math. Acad. Sinica, 33 (2005), 77-90.

[8] S. Deshmukh, B. Y. Chen, S. Alshamari, On rectifying curves in Euclidean 3-space, Turk. J. Math., 42 (2018), 609-620.

[9] K. Ilarslan, E. Nešović, Timelike and null normal curves in Minkowski space $\mathbb{E}_{1}^{3}$, Indian J. Pure Appl. Math., 35(7) (2004), 881-888.

[10] K. Ilarslan, E. Nešović, On rectifying curves as centrodes and extremal curves in the Minkowski 3-Space, Novi Sad J. Math., 37 (2007), 53-64.

[11] K. Ilarslan, E. Nešović, T. M. Petrović, Some characterization of rectifying curves in the Minkowski 3-Space, Novi Sad J. Math., 33 (2003), $23-32$.

[12] R. Lopez, Differential Geometry of curves and surfaces in Lorentz-Minkowski space, Int. Electron. J. Geom., 7(1) (2014), 44-107.

[13] B. O’Neill, Semi-Riemannian Geometry with Applications to Relativity, Academic Press, New York, 1983. 